\title{
Experimental Research of Wire Cut EDM for SR \& MRR using Taguchi Method
}

\section{A. Raveendra}

\begin{abstract}
The present research work is to examine \& advance the latent procedure factors affecting the MRR, SR and Electrode Attire despite the fact machining of Nickel composites utilizing WEDM progression. This exertion includes investigation of the connection amid the different information route considerations like Pulse-on time(Ton), Pulse off time(Toff), Pulse Peak Current(IP), Wire substantial, Work piece material \& procedure factors. In light of the picked information parameters and execution estimates L-16 symmetrical exhibit is chosen to streamline the most appropriate qualities for machining for nickel amalgams by WEDM...
\end{abstract}

Keywords: Surface Quality; EDM; Taguchi Method;MRR.

\section{INTRODUCTION}

Wire Cut Electric Discharge Machining process with a slight wire as a cathode changes electrical vitality to warm vitality for cutting materials, WEDM is considered as an interesting reception of the customary EDM process, which utilizes a cathode to instate the starting procedure [1]. The assortments of crop rejoinders with route strictures were numerically confirmed by utilizing non-direct deterioration examination [2]. The models were checked for their ampleness. Consequence of affirmation tests demonstrated that the set up scientific models can foresee the yield reactions with sensible exactness [3]. Be that as it may, WEDM uses a constantly voyaging wire anode made of slender copper, metal or tungsten of breadth $0.05-0.30 \mathrm{~mm}$, which is equipped for accomplishing extremely little corner radii [4-6]. The wire is kept in strain utilizing a mechanical tensioning gadget diminishing the inclination of creating wrong parts [5-7]. During the WEDM procedure, the material is dissolved in front of the wire and there is no immediate contact between the work piece and the wire, wiping out the mechanical worries during machining $[8,9]$.

\section{EXPERIMENTAL PROCEDURE}

Examinations have been performed so as to explore the impacts of at least one elements of the procedure parameters superficially finish of the wire cut machined surface. Fig 1 to

Revised Manuscript Received on December 30, 2019.

* Correspondence Author

A.Raveendra, Department of Mechanical Engineering, Malla Reddy Engineering, College (Autonomous), Hyderabad, Telangana 500100 India. E-mail: ravi.akunuru@gmail.com;Mob: 9502294258.

(C) The Authors. Published by Blue Eyes Intelligence Engineering and Sciences Publication (BEIESP). This is an open access article under the CC BY-NC-ND license (http://creativecommons.org/licenses/by-nc-nd/4.0/)
8 shows the EDM machine which comprises the input variables and taguchi design and analysis and their process parameters.

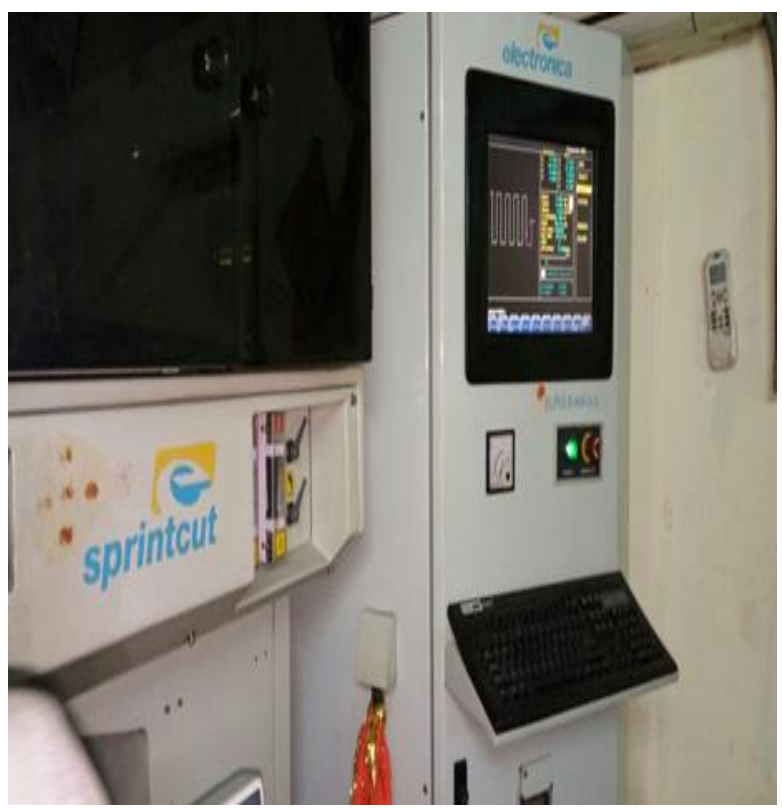

Fig 1 Control panel of EDM.

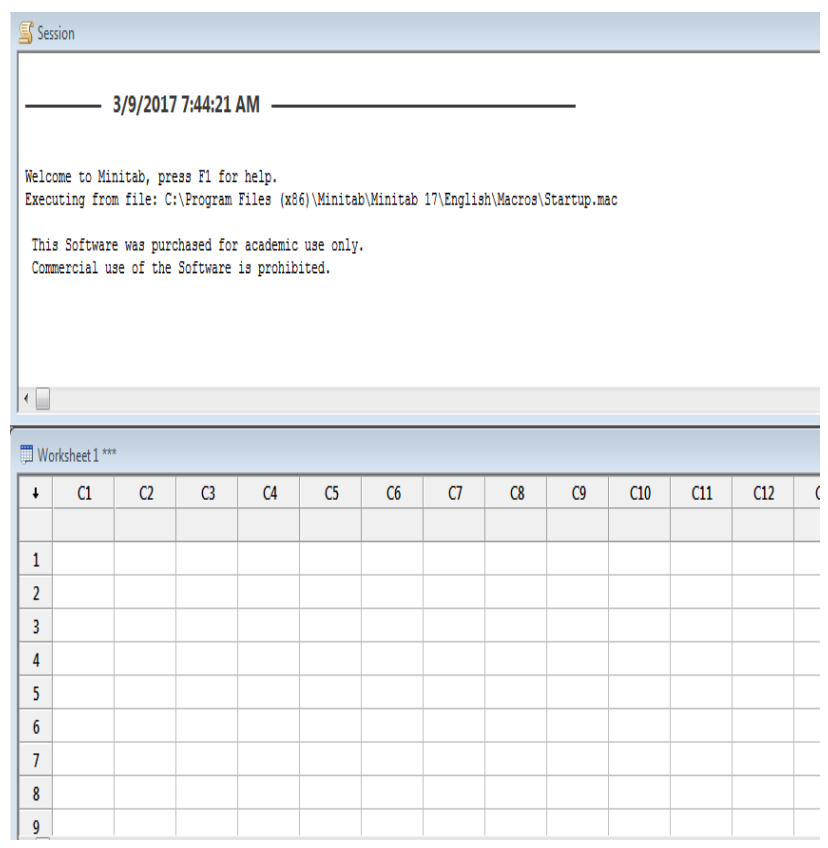

Fig 2 Input screen of EDM.

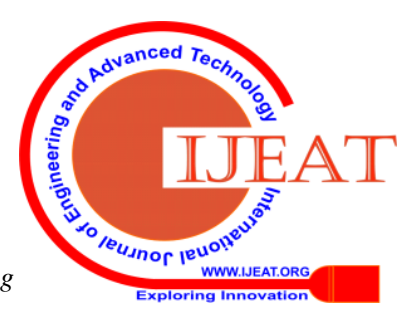




\section{Experimental Research of Wire Cut EDM for SR \& MRR using Taguchi Method}

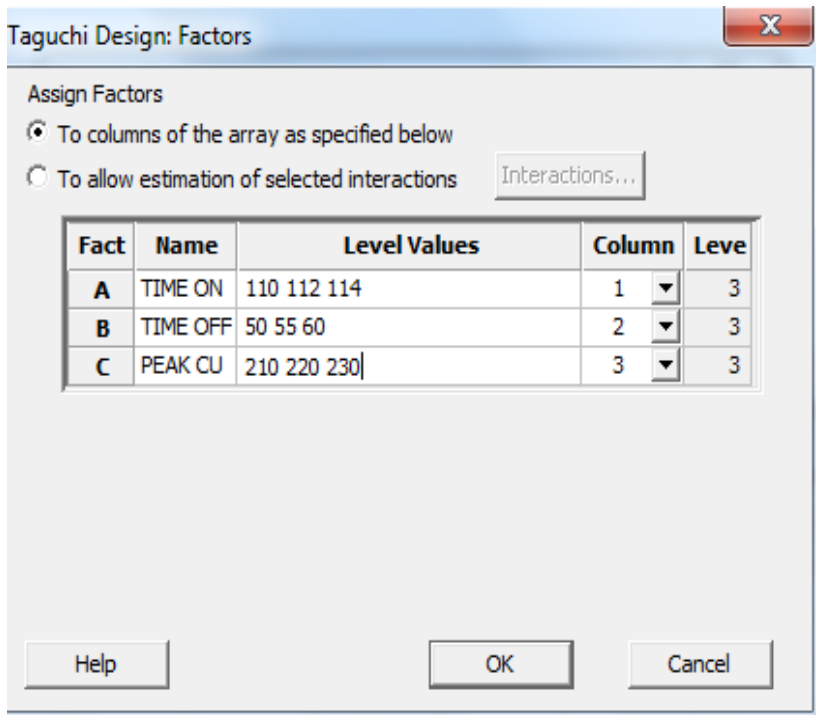

Fig 3 Display Available Designs Select - L9 (2-4)

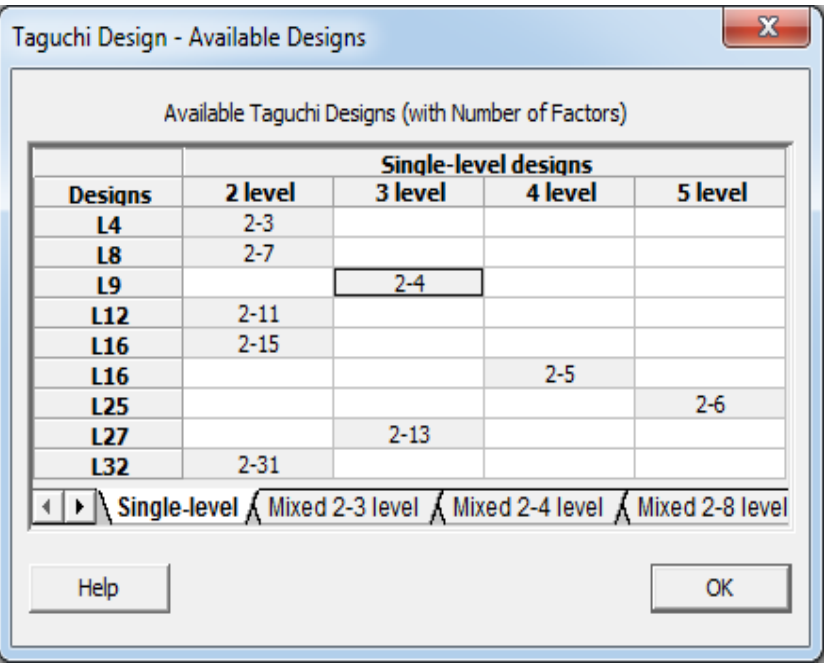

Fig 4 Select Designs Select - L9

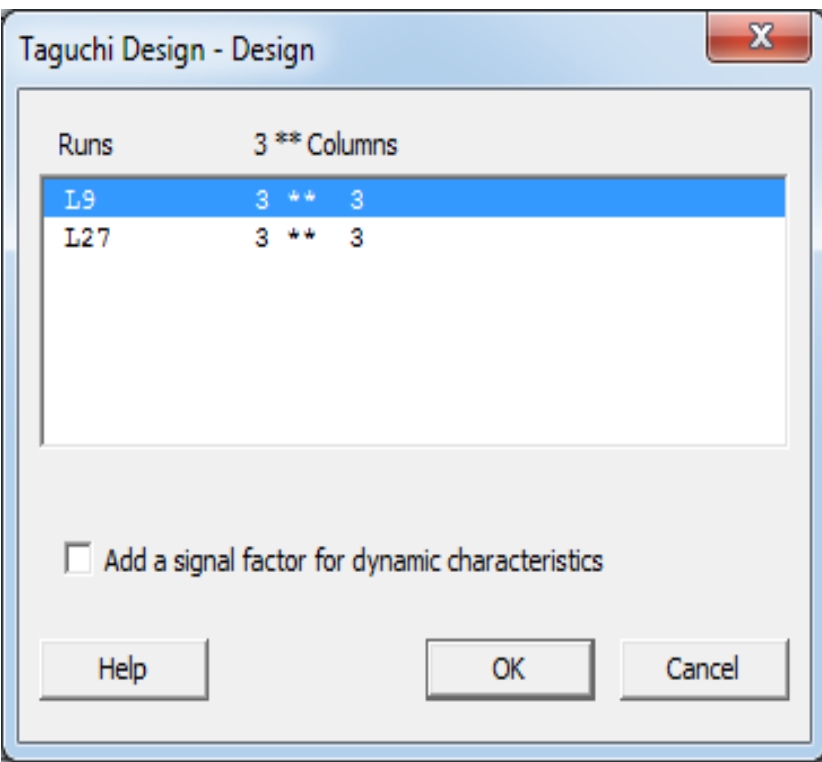

\begin{tabular}{|c|c|c|c|c|c|}
\hline \multicolumn{6}{|c|}{ Worksheet 2 *** } \\
\hline$\downarrow$ & $\mathrm{C} 1$ & $\mathrm{C} 2$ & $\mathrm{C} 3$ & $\mathrm{C4}$ & $\mathrm{C5}$ \\
\hline & TIME ON & TIME OFF & PEAK CURRENT & SURFACE FINISH & SURFACE FINISH 1 \\
\hline 1 & 110 & 50 & 210 & 0.30 & 0.31 \\
\hline 2 & 110 & 55 & 220 & 0.36 & 0.38 \\
\hline 3 & 110 & 60 & 230 & 0.41 & 0.40 \\
\hline 4 & 112 & 50 & 220 & 0.33 & 0.32 \\
\hline 5 & 112 & 55 & 230 & 0.39 & 0.37 \\
\hline 6 & 112 & 60 & 210 & 0.45 & 0.46 \\
\hline 7 & 114 & 50 & 230 & 1.04 & 1.02 \\
\hline 8 & 114 & 55 & 210 & 0.88 & 0.86 \\
\hline 9 & 114 & 60 & 220 & 0.94 & 0.96 \\
\hline
\end{tabular}

Fig 5 Taguchi - Analyze Taguchi Design - Select Responses

\begin{tabular}{|c|c|c|c|}
\hline \multicolumn{4}{|c|}{ Worksheet 2 . } \\
\hline+ & $\mathrm{C} 1$ & $\mathrm{C} 2$ & $\mathrm{C} 3$ \\
\hline & TIME ON & TIME OFF & PEAK CURRENT \\
\hline 1 & 110 & 50 & 210 \\
\hline 2 & 110 & 55 & 220 \\
\hline 3 & 110 & 60 & 230 \\
\hline 4 & 112 & 50 & 220 \\
\hline 5 & 112 & 55 & 230 \\
\hline 6 & 112 & 60 & 210 \\
\hline 7 & 114 & 50 & 230 \\
\hline 8 & 114 & 55 & 210 \\
\hline 9 & 114 & 60 & 220 \\
\hline
\end{tabular}

\section{Analyze Taguchi Design}

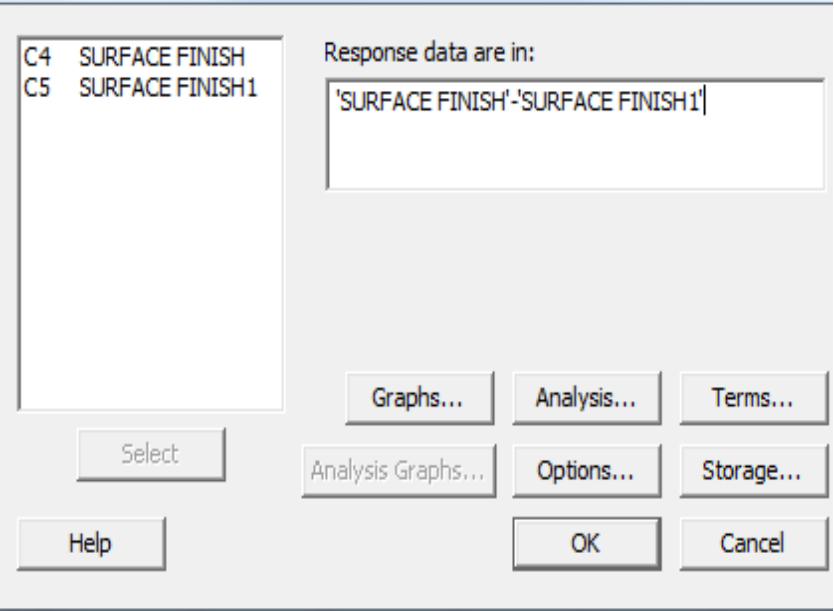

Fig 6 Taguchi Design
Published By:

Blue Eyes Intelligence Engineering \& Sciences Publication 


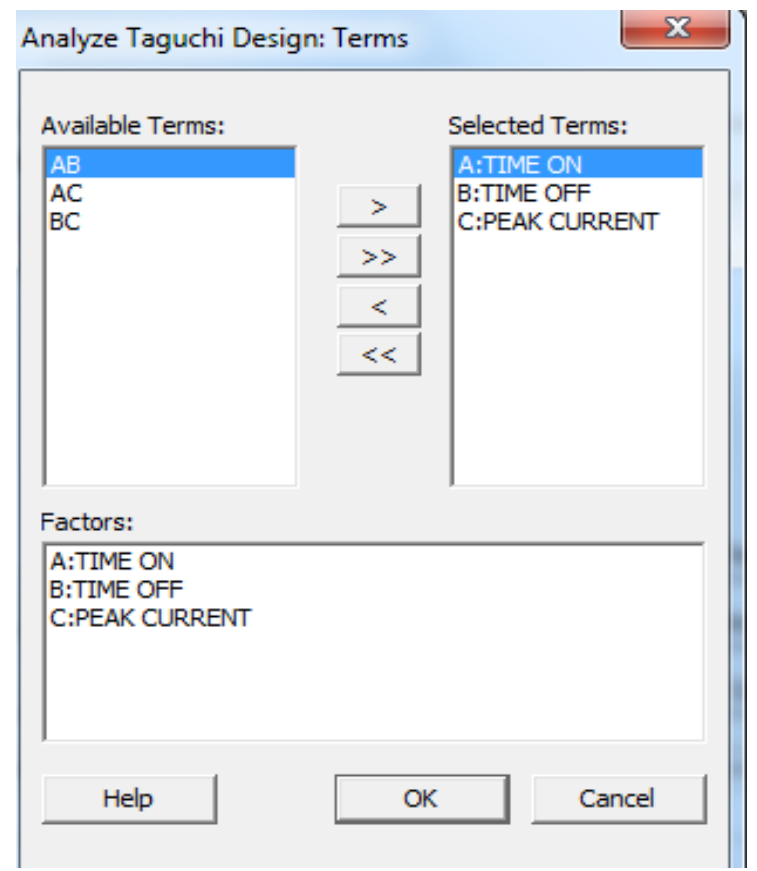

Fig 7 Taguchi Design

Analyze Taguchi Design - Analysis
\begin{tabular}{|l|l|}
\hline Display response tables for & Fit linear model for \\
$\sqrt{\nabla}$ Signal to Noise ratios & $\Gamma$ Signal to Noise ratios \\
$\nabla$ Means & $\Gamma$ Means \\
$\Gamma$ Standard deviations & $\Gamma$ Standard deviations \\
\hline Help & OK \\
\hline
\end{tabular}

Fig 8 Taguchi Analysis

Table 1. Input Factor

\begin{tabular}{|c|c|c|c|c|}
\hline \multirow[t]{2}{*}{ Experiments } & \multicolumn{2}{|c|}{ Time } & \multirow{2}{*}{$\begin{array}{c}\text { Peak } \\
\text { current } \\
\text { (IP) }\end{array}$} & \multirow{2}{*}{$\begin{array}{c}\text { Voltage } \\
\text { (V) }\end{array}$} \\
\hline & On & Off & & \\
\hline 1 & 108 & 62 & 160 & 23 \\
\hline 2 & 109 & 61 & 170 & 23 \\
\hline 3 & 110 & 60 & 180 & 23 \\
\hline 4 & 111 & 59 & 190 & 23 \\
\hline 5 & 112 & 58 & 200 & 25 \\
\hline 6 & 113 & 57 & 210 & 26 \\
\hline 7 & 114 & 56 & 220 & 27 \\
\hline 8 & 115 & 55 & 230 & 28 \\
\hline 9 & 116 & 54 & 230 & 30 \\
\hline
\end{tabular}

Table 2 . The 19 Orthogonal Array For Input Parameters Pulse On Time, Pulse Off Time And Peak Current

\begin{tabular}{|c|c|c|c|}
\hline \multirow{2}{*}{ Experiments } & \multicolumn{2}{|c|}{ Time } & $\begin{array}{c}\text { Peak } \\
\text { current (IP) }\end{array}$ \\
\cline { 2 - 3 } & On & Off & 210 \\
\hline 1 & 110 & 50 & 220 \\
\hline 2 & 110 & 55 & 230 \\
\hline 3 & 110 & 60 & 210 \\
\hline 4 & 112 & 50 & 220 \\
\hline 5 & 112 & 55 & 230 \\
\hline 6 & 112 & 60 & 210 \\
\hline 7 & 114 & 50 & 220 \\
\hline 8 & 114 & 55 & 230 \\
\hline 9 & 114 & 60 & \\
\hline
\end{tabular}
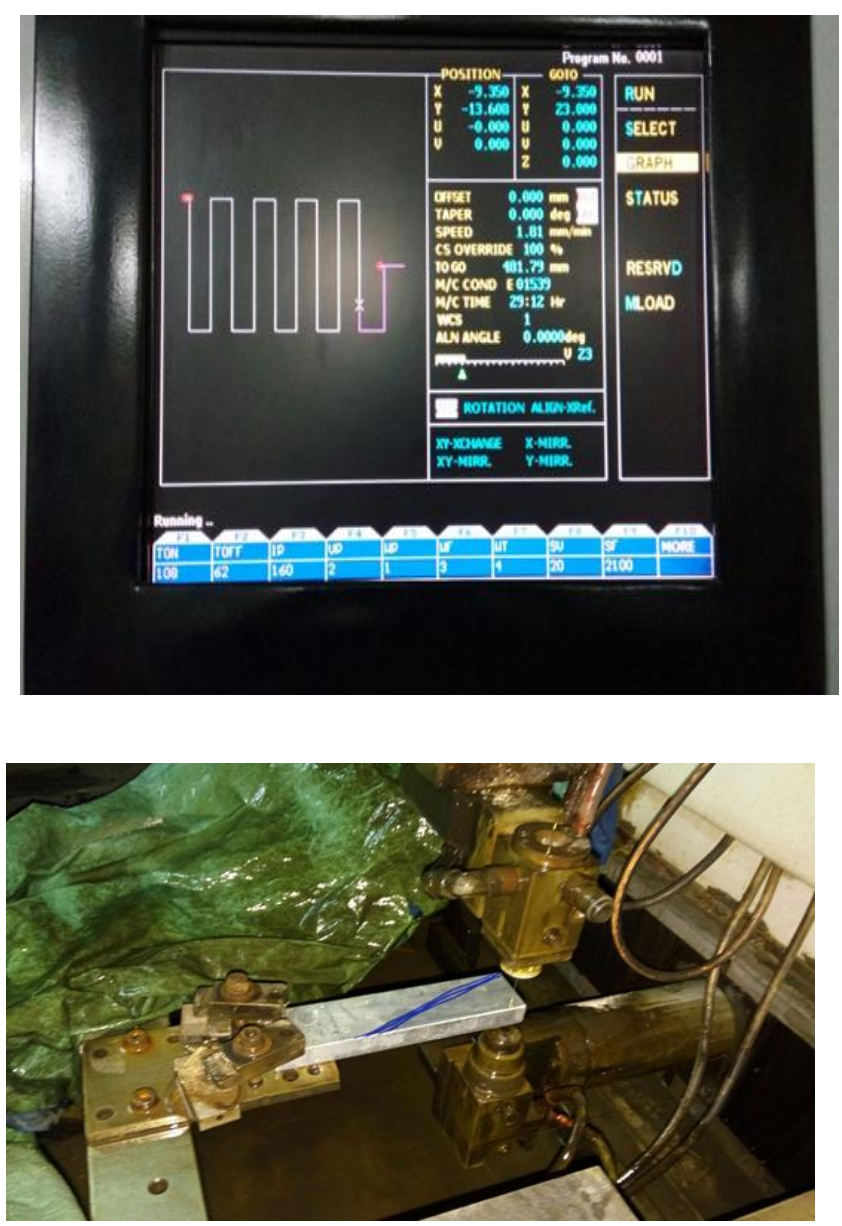

\section{RESULTS}

Taguchi technique focuses on the significance of examining the reaction variety utilizing the sign to-commotion $(\mathrm{S} / \mathrm{N})$ proportion, bringing about minimization of value trademark variety because of wild consideration. 


\section{Experimental Research of Wire Cut EDM for SR \& MRR using Taguchi Method}
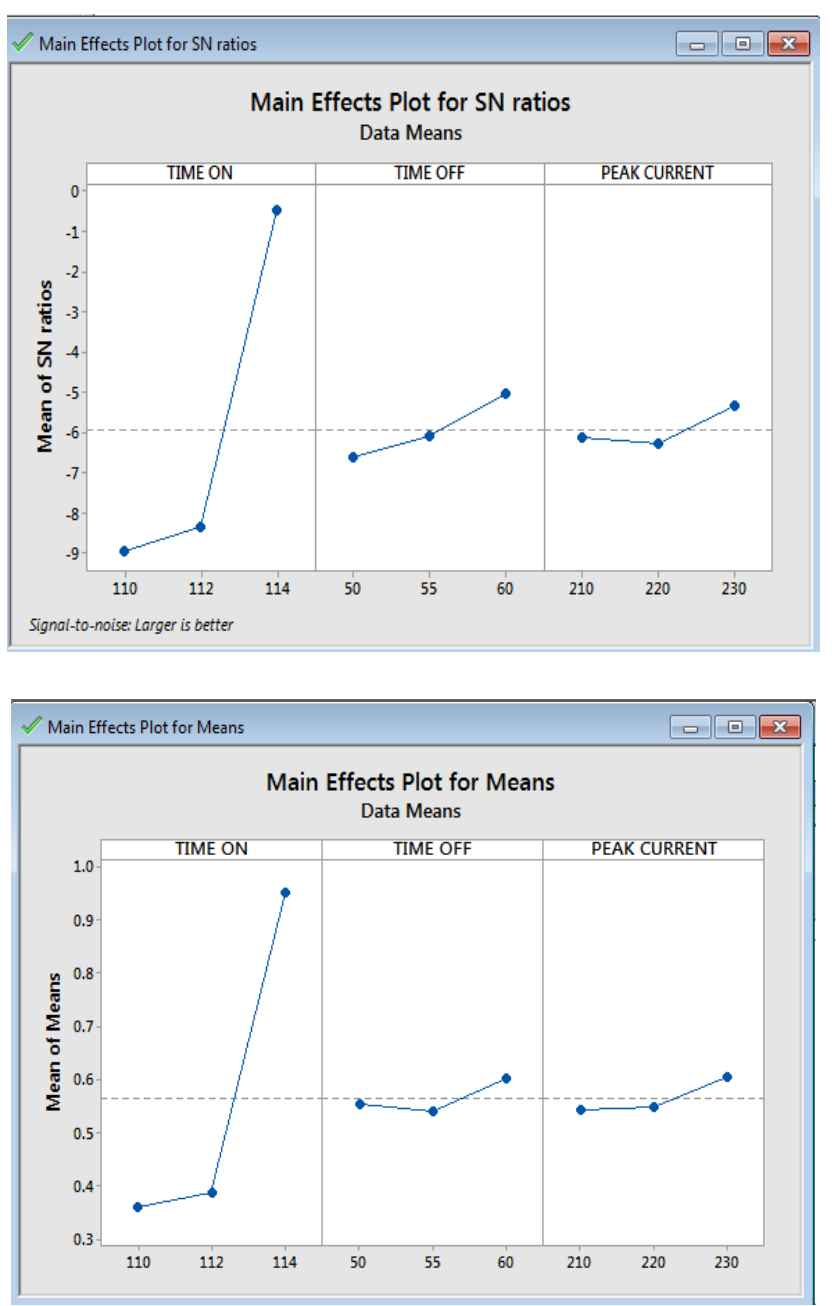

Table 3 Surface Finish Results

\begin{tabular}{|c|c|c|c|c|}
\hline \multirow{2}{*}{ Experiments } & \multicolumn{2}{|c|}{ Time } & $\begin{array}{c}\text { Peak } \\
\text { curent } \\
\text { (IP) }\end{array}$ & Surface Finish Values $\mathrm{R}_{\mathrm{a}}$ \\
\cline { 2 - 5 } & On & Off & & \\
\hline 1 & 110 & 50 & 210 & 0.3 \\
\hline 2 & 110 & 55 & 220 & 0.36 \\
\hline 3 & 110 & 60 & 230 & 0.41 \\
\hline 4 & 112 & 50 & 210 & 0.33 \\
\hline 5 & 112 & 55 & 220 & 0.39 \\
\hline 6 & 112 & 60 & 230 & 0.45 \\
\hline 7 & 114 & 50 & 210 & 1.04 \\
\hline 8 & 114 & 55 & 220 & 0.88 \\
\hline 9 & 114 & 60 & 230 & 0.94 \\
\hline
\end{tabular}

\section{CONCLUSION}

The target of the contemporary effort is to research the impacts of the different Wirecut EDM progression factors on the machining eminence \& the ideal arrangements of procedure factors consequently the nature of machined fragments can be enhanced. Analyses are led on the pieces changing restrictions. The materials utilized for machining are Aluminum combination. Streamlining is finished utilizing Minitab programming..

\section{REFERENCES}

1. M. D. Vijayakumar, et.al., Experimental investigation on single point incremental forming of IS513Cr3 using response surface method, Materials Today: Proceedings.
2. T. Adithiyaa et.al.,, Optimal Prediction of Process Parameters By GWO-KNN in Stirring-Squeeze Casting of AA2219 Reinforced Metal Matrix Composites, Materials Today: Proceedings (2019). DOI:10.1016/j.matpr.2019.10.051.

3. Chandramohan, D et al.. Journal of Bio- and Tribo-Corrosion (2019) 5:66.DOI: https://doi.org/10.1007/s40735-019-0259-z

4. K Gurusami, et.al. (2019): Int. J. Amb. Energy, DOI: 10.1080/01430750.2019.1614987.

5. Sathish, T., Chandramohan, D. International Journal of Recent Technology and Engineering,7(6), 287-290,2019.

6. Chandramohan, D., Rajesh, S. Acad. J. of Mfg. Eng.,12(3),67-71,2014

7. Sathish, $\mathrm{T}$ and Chandramohan, $\mathrm{D}$, Teaching methods and methodologies used in laboratories, International Journal of Recent Technology and Engineering Volume 7, Issue 6, March 2019, Pages 287-290.

8. Sathish,T. et.al., International Journal of Mechanical and Production Engineering Research and Development, Volume 2018, Issue Special Issue, 2018, Article number IJMPERDSPL201883, Pages 705-710.

9. Chandramohan, D., Rajesh, S. Acad. J. of Mfg. Eng.,12(3),72-77,2014

10. Sathish, T., Chandramohan, D., International Journal of Recent Technology and Engineering,7(6), 281-286,2019.

\section{AUTHORS PROFILE}

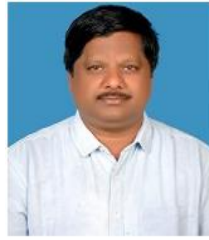

Dr.A.Raveendra, Associate Professor and Head of the Department of Mechanical Engineering, Malla Reddy Engineering college (Autonomous),Maisammaguda, Secunderabad. He has 17 years of teaching and 6 years industry experience. His B.Tech degree is from REC -Warangal and M.Tech(Production Engg)from VTU-Belgaum. He has published 35 papers in various International journals and presented 18 papers in International conferences. He has guided 40 B.Tech projects and 21 M.Tech projects. He is the life member of ISTE, IWS, IAENG. 